\title{
Kinematic cues and recognition of self-generated actions
}

\author{
Elena Daprati • Selina Wriessnegger • \\ Francesco Lacquaniti
}

Received: 20 April 2006 / Accepted: 21 July 2006 / Published online: 22 August 2006

(C) Springer-Verlag 2006

\begin{abstract}
In the present study, we addressed the issue of whether healthy individuals can recognize a given gesture as their own, based on kinematic information. To this purpose, we required 36 volunteers to execute a series of hand movements of increasing complexity, while their kinematics was recorded by a motion-capture system. In a later session, we showed them a series of computer animations where a virtual hand, rendered as a simple stick-diagram, was animated by the kinematics recorded from the participants in the previous session. Their task was to recognize their own movements, choosing from three alternatives. To test the contribution of various potential cues to action recognition, the roles of (1) access to motor representation, (2) gesture complexity, and (3) familiarity effects were separately investigated. The results support the hypothesis that kinematic templates rather than single
\end{abstract}

E. Daprati $(\varangle) \cdot$ S. Wriessnegger $\cdot$ F. Lacquaniti

Department of Neuromotor Physiology,

IRCCS Santa Lucia Foundation,

Via Ardeatina 306, 00179 Rome, Italy

e-mail: e.daprati@hsantalucia.it

S. Wriessnegger

Department of Psychology, Max-Planck-Institute for Human

Cognitive and Brain Sciences, Amalienstrasse 33,

80799 Munich, Germany

F. Lacquaniti

Department of Neuroscience,

University of Rome Tor Vergata, Via Montpellier 1,

00133 Rome, Italy

F. Lacquaniti

Center of Space BioMedicine,

University of Rome Tor Vergata, Via O. Raimondo 8,

00173 Rome, Italy motor parameters contribute to self-recognition in the absence of morphological cues.

Keywords Action recognition · Self ·

Motor representation $\cdot$ Biological motion .

Motor imagery $\cdot$ Action styles

\section{Introduction}

In the past few years, several attempts have been made to study the mechanisms that enable us to correctly identify self-generated actions. Two main approaches have been followed: either the origin of the action was made ambiguous within a real-time setting, or multiple alternatives for the observer's action were provided in delayed paradigms.

Real-time paradigms use an experimental setting where an action is actively executed and visually monitored, but its origin is purposefully made ambiguous (Nielsen 1963; Daprati et al. 1997; Franck et al. 2001). In one version (Daprati et al. 1997), upon hearing a verbal command, healthy volunteers executed a particular gesture with their unseen gloved hand. Simultaneously, the image of a moving, similarly gloved hand was presented on a screen in front of them. Participants were asked to decide whether the movement projected on the screen was their own. Ambiguity was introduced by capturing the movement of the participant's hand as well as that of the hand of a hidden actor, with two separate cameras. By means of an experimenter-controlled switch, either hand could appear on the screen in front of the observer. In this condition, efferent information-from volition to motor plans-was entirely available to the participants, and could be integrated with 
visual feedback and afferent information from the moving limb, to guide their responses. In this experiment, participants were good at recognizing their movements, detecting their gesture in approximately $70 \%$ of trials (Daprati et al. 1997). Interestingly, mistakes were almost entirely attributions of the actor's movements to the self, suggesting that signals arising from kinesthetic feedback could be overridden by other cues. A later study confirmed that if the alien movement (1) corresponded to the observer's intentions, (2) started in synchrony with that of the observer, and (3) took place in the expected location, the small kinematic differences that characterize individuals were easily ignored (Franck et al. 2001). Within this framework, it has been proposed that the ability to recognize a movement as one's own largely relies on the opportunity to process efferent information about motor production (i.e., volitional and intentional information; motor planning). Furthermore, it necessarily depends on the capacity to continuously monitor motor execution (for a review, see Daprati and Sirigu 2005).

The major drawback of real-time settings resides in the possible role that volitional and anticipatory cues might play in self-recognition. Indeed, availability of efferent cues leads to more accurate movement attribution (Tsakiris et al. 2005), and may account for the fact that errors are most likely to occur as attribution of other's movement to the self, rather than the opposite. Separating action execution and action recognition in time can largely reduce this eventuality. This approach has been widely used following the seminal work by Wolff (1943) and Johansson (1973) on recognition of biological motion. Johansson's study showed that observers could recognize a human walker in extremely impoverished settings such as when a point-light display was the only available information. Interestingly, in these conditions, observers are able to identify gender (Kozlowski and Cutting 1977) and even identity (Beardsworth and Buckner 1981) of the moving actor. To date, researchers using delayed paradigms in self-recognition studies have concentrated mostly on the optical (Knoblich and Prinz 2001; Loula et al. 2005) or acoustical traces of movements (Flach et al. 2004; Repp and Knoblich 2004). In each case, subjects' accuracy was comparable to that seen in real-time settings. Although neither approach directly explored the parameters involved in recognition processes, the results suggest a strong involvement of movement-related cues. For instance, it was shown that removal of important signatures of biological motion, such as velocity profiles, dramatically reduced accuracy (Knoblich and Prinz 2001). Similarly, in individuals whose ability to monitor motor execution is defective, as can be the case following left parietal lesion, attribution errors were significantly increased (Sirigu et al. 1999).

The present research aims at better defining the nature of the motor-related cues involved in self-identification. Three kinds of information, readily made available by voluntary motor activity, were investigated here: (a) first-person efferent information (i.e., intention to move, motor planning, mental representation of the motor act), (b) individual characteristics of one's kinematics, and (c) visual familiarity with one's movements. The differential role of these features in self-identification was assessed in separate experiments. In particular, in Experiment 1, we used a delayed paradigm to separate in time the processes of motor execution and action recognition. Furthermore, we asked participants to reactivate first-person efferent information through mental rehearsal at the time of action recognition, thus isolating this component from signals derived from volition and/ or motor-related sensory reafferences that are specific to real-time action. In Experiment 2, we separated motion cues from morphological cues arising from the hand's shape and size, by using a virtual hand animated by the participant's own kinematics. We also selected gestures of increasing complexity to allow for differential expression of individual differences in execution. More particularly, we varied the contribution of three major motion features, i.e., rhythm, degree of fingers' independence, and familiarity of the hand posture. Finally, in Experiment 3, we assessed the contribution to self-identification of visual familiarity with one's actions. For this purpose, in separate groups of participants, we either prevented participants from observing their actions during execution, or manipulated the visual output of the movement.

\section{Experiment 1}

In Experiment 1, we assessed the role of first-person efferent information in identification of ones' own motion pattern. In particular, we tested whether reactivation of volitional processes, and/or mechanisms related to motor planning, can facilitate detection of one's own gesture, in the absence of overt action execution. This was done by engaging participants in a motor imagery task.

\section{Methods}

\section{Participants}

Twenty right-handed volunteers (12 females, 8 males; mean age $23.8 \pm 4.9$ years; education $15.3 \pm 1.4$ years) 
were recruited among students from local university and nursing schools, and gave informed consent to participate (as required by the local ethics guidelines, which comply with the 1964 Declaration of Helsinki). They had normal or corrected to normal vision and did not report previous history of neurological or psychiatric disorders. All were right-handed according to a shortened version of the Edinburgh Inventory (mean laterality ratio $0.7 \pm 0.2$; Oldfield 1971). In order to obtain a measure of these subject's imagery abilities, the Movement Imagery Questionnaire (MIQ, Hall and Pongrac 1983) was administered to the participants. The MIQ is a self-reporting questionnaire requiring subjects to rate their difficulty in imagining movements on a 7-point scale: the lower their score the higher their movement imagery ability (10 or less $=$ high, 11$22=$ medium, above 23 =low; Corlett et al. 1989). Here, six participants scored as medium imagers (mean $18.3 \pm 3.8$ ), and eight were low imagers (mean score $30.3 \pm 6.6$ ). Six participants completed only part of the questionnaire due to time constraints, scoring as medium to low imagers.

\section{Procedure}

The experiment was run in two separate sessions. In session 1, participants were asked to execute hand gestures. In session 2, they were required to identify their gestures among alternatives. No reference was made in session 1 to the fact that the next session would require self-recognition of the presently executed movements. This was done to limit the possibility that participants activated overt encoding strategies or were less spontaneous in their execution.

\section{Session 1}

Participants sat in front of a table $(50 \times 60 \times 85 \mathrm{~cm})$, and positioned their right hand on its surface in a fixed location (starting position). The experimenter showed participants examples of six hand gestures, which they later executed, one at a time, on verbal command. Each gesture started and ended with the hand flat on the table in the starting position. Although a model of the gesture was provided, participants were asked to execute each movement at their own pace, trying to act 'as spontaneously as possible'. Gestures were: 'tapping with the hand' (HT, hand taps three times); 'tapping with the fingers' (FT, index, middle, ring and little finger tap once, individually); ' walk' with two fingers' (FW, index and middle finger move four 'steps'); 'trace a line' (TL, thumb and index join to grasp an imaginary piece of chalk, trace a line of desired length and orien- tation). Two additional gestures were included, 'draw a circle' and 'write a word' (i.e., 'lago', lake in Italian), which acted as fillers and were not used in the following session. The experimenter initiated each trial by telling the participant which movement he/she was supposed to execute. Once the gesture had been produced, the experimenter moved on to the next. Fifteen repetitions of each gesture were required, in pseudo-random order. The temporal and spatial parameters of the movements were recorded by a motion capture system (Vicon Motion Systems Ltd., UK). The system includes nine TV-cameras detecting infrared reflecting markers at the sampling rate of $100 \mathrm{~Hz}$, with a spatial accuracy of approx. $1 \mathrm{~mm}$. Twenty-five markers $(\varnothing$ $2 \mathrm{~mm}$ ) were placed on the back of the subjects' right upper limb, as shown in Fig. 1a. At the end of the recording session, the time-course of the $3 \mathrm{D}$ locations of each marker was reconstructed by dedicated software. Animation data were exported and associated with a $3 \mathrm{D}$ model of a hand (see Fig. 1b) whose elements were white sticks ( $\varnothing 15 \mathrm{~mm})$ linking the main hand joints (model derived from Vicon Polygon Visualization Tool). The virtual hand maintained the original proportions of the real hand (e.g., phalange length, wrist width) of each participant. For each gesture, eight repetitions (free from procedural or recording errors) were reconstructed. Separate video-clips, each lasting approximately $3 \mathrm{~s}$, were created for each movement.

\section{Session 2}

Self-recognition was tested 7-8 days after the recording session. Participants sat in a dimly lit room, at about $50 \mathrm{~cm}$ from a PC-screen $(40 \times 30 \mathrm{~cm})$, their upper limbs resting on their thighs. In each trial, three videos appeared in brief sequence on the screen. Each video showed the virtual hand, displayed on a black background. The hand appeared in the lower left quadrant of the screen, to roughly match the position in the subject's visual field during the recording session, and occupied an area of about $8 \times 6 \mathrm{~cm}$. One trial consisted of three videos presenting the same gesture, executed by the observer and two other participants (distractorparticipants). Both distractor-participants had the same the sex as the observer and were kept constant for the entire session, so that gestures from three different persons occurred with the same frequency during the task. The observer was required to carefully watch each movement and to choose the video showing her/his own gesture. No information was given about number or sex of the individuals acting as distractors. The timing of the events is shown in Fig. 1c. Time limits were not imposed. The experimenter manually recorded 

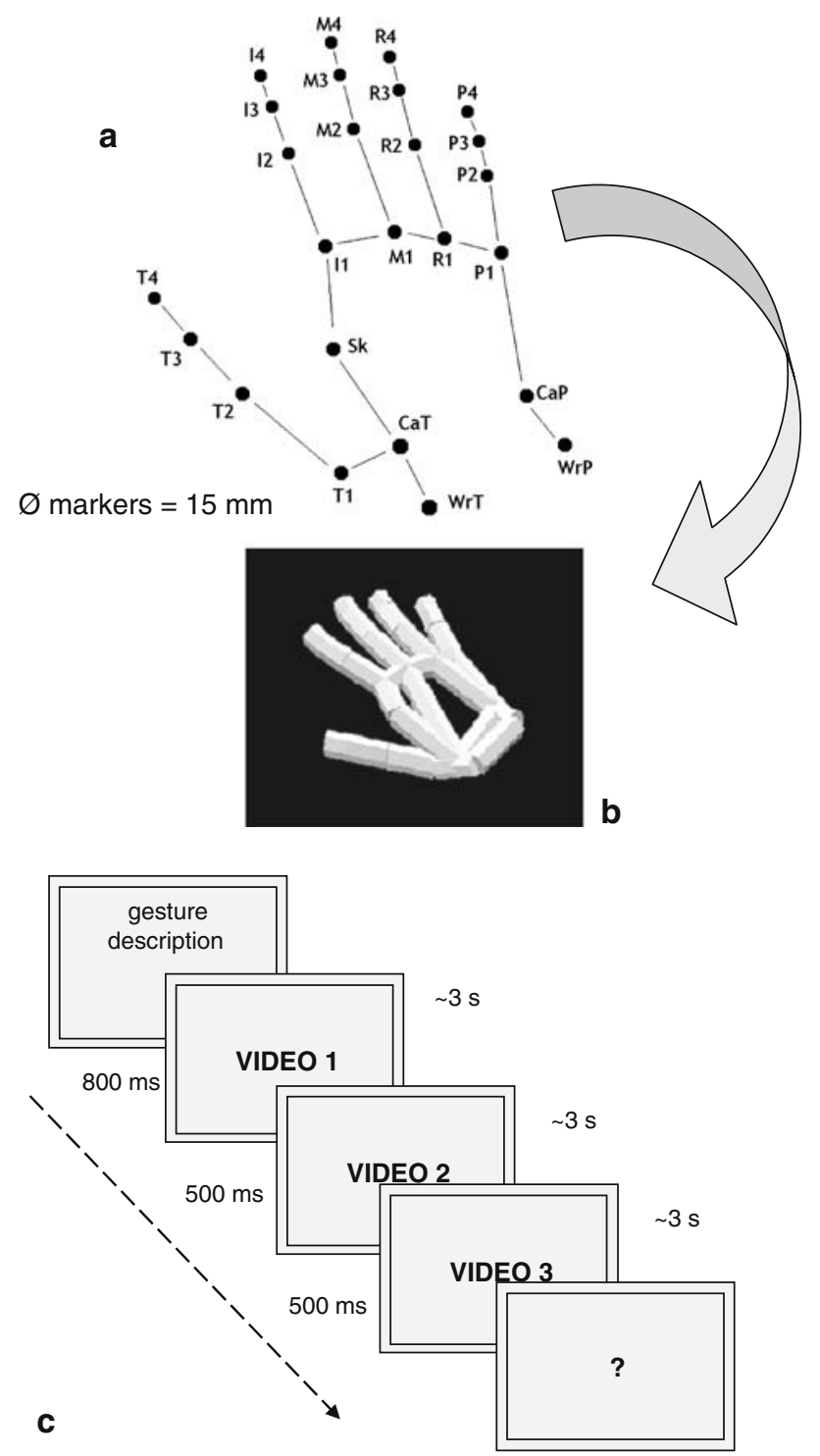

Fig. 1 a Marker locations in the recording session. Twenty-five reflecting markers were placed on the subjects' dominant hand, as follows: two markers were placed on the distal forearm (styloid process of the radius and head of the ulna, $\mathrm{WrT}, \mathrm{WrP}$ ) and two markers were placed on the wrist (carpus proximal bones, thumb and pinkie side $\mathrm{CaT}, \mathrm{CaP}$ ). Four markers were placed on the thumb, at the level of the 1st metacarpal base, 1st metacarpal head, interphalangeal joint and finger-tip (T1, 2, 3, 4); similarly, four markers were placed on each of the remaining four fingers, at the level of the metacarpal head, proximal interphalangeal joint, distal interphalangeal joint, and finger-tip (I, M, R, P1-4). Finally, one marker was placed on the skin, at the mid-point between the heads of the 1st and 2nd metacarpal bones (Sk). b Example of the virtual hand used in Experiment 1. c Schematic description of the event sequence in the recognition session (more details in text)

responses. Eight sequences of three videos were presented for each gesture, in four short blocks of eight trials each. Videos corresponding to the subjects' gestures occurred with equal probability in the first, second and third place, as did those of the two distractor-participants. The first block was repeated at the end of the series to check for consistency in the responses and/or learning effects.

\section{Design}

Participants were randomly assigned to two equal groups, which did not differ in terms of the major demographic measures (age, education, laterality ratio). Participants in Group 1 (Imagery) were advised to mentally rehearse the four movements prior to each experimental block (i.e., before every ninth trial). In particular, they were asked to close their eyes and, following a go-signal, mentally simulate a given gesture. They opened their eyes as soon as they finished the imagery task. Mental imagery was performed at least twice for each gesture, prior to each block. To make sure that participants accomplished the task, one experimenter verified with a stopwatch that the time to mentally complete each movement was roughly comparable to the mean execution time in the recording session. To further stress use of imagery during the task, we invited participants to mentally rehearse the gesture before giving their answer in each trial. Participants in Group 2 (Control) did not receive any specific instruction prior to each experimental block. At the end of the experiment (total duration of 20-25 min), all participants received a questionnaire asking them to rate task difficulty on a 7-point scale, report strategies used to solve the task, and comment on several methodological aspects (i.e., number of persons whose movements were presented, their sex, etc.). One subject in the Control group could not return to the lab for the second session and was thus discarded from the study.

\section{Data analysis}

For each gesture, the proportion of correctly recognized movements was computed for all participants. Trials corresponding to errors in the procedure during stimulus presentation were discarded from the analysis (less than 1\%). Consistency in the responses for Block 1 during the first and second presentation was used to assess subjects' reliability. Means of correct responses for each group were compared by means of a 2-way ANOVA where Group (Imagery vs. Control) was a between-subject variable and Gesture (four levels: HT, FT, FW, TL) was a within-subject variable. In order to control for the effects of a skewed distribution and satisfy the conditions for parametric statistical test, the proportions of correct responses were first submitted 
to an arcsine transformation. A Scheffè test was used for post-hoc analyses.

Results

\section{Accuracy}

A 2-way ANOVA comparing the two groups of participants showed only a significant main effect of Group $\left(F_{(1,17)}=6.73 ; P<.02\right)$. Subjects in the Control group correctly chose the video corresponding to their movement in about $65 \%$ of trials (overall proportion of correct detections and $\mathrm{SD}, 0.65 \pm 0.18$ ), whereas subjects performing mental rehearsal prior to recognition (Imagery group) correctly detected their movement in approximately $75 \%$ of trials (overall proportion of correct detections $0.76 \pm 0.25$ ). The main effect of gesture was non-significant, as was the interaction.

Within the Control group, responses were significantly above chance for all gestures, but the proportion of correct identifications seemed to vary according to gesture. Although the effect was not statistically significant, a slightly higher recognition rate was found for 'tapping with the finger' (FT, $0.78 \pm 0.18$ ), compared to the other gestures $(\mathrm{HT}, 0.69 \pm 0.35$; FW, $0.61 \pm 0.24$; TL, $0.52 \pm 0.32$, see also Fig. 2). This tendency was not found in the Imagery group (TL: $0.82 \pm 0.22$; FW: $0.74 \pm 0.25$; HT: $0.69 \pm 0.27$; FT: $0.76 \pm 0.28$, see also Fig. 2). As suggested from mean values, responses were significantly above chance for all gestures (TL: $\chi^{2}=62.79 ; \quad$ FW: $\quad \chi^{2}=44.92 ; \quad$ HT: $\quad \chi^{2}=54.42 ; \quad$ FT: $\chi^{2}=48.17$, all $P<0.001$ ).

The data were further explored to exclude biases due to a progressive learning of the task, or to the relative position (1st, 2nd, 3rd), which the observer's

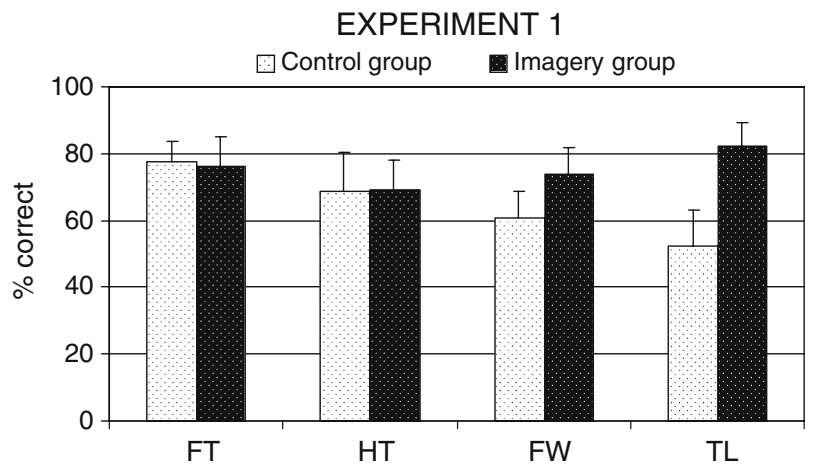

Fig. 2 Percentage of correctly recognized gestures for the movements tested in Experiment 1. Gray bars refer to subjects in the Control group, black bars to subjects in the Imagery group. Whiskers report standard error of the mean. Abbreviations on $x$-axis indicate gestures: $F T$ tapping with the fingers, $H T$ tapping with the hand, $F W$ walk with two fingers, $T L$ trace a line movement (target-video) occupied in the series. In both the Control and the Imagery group, learning effects were not apparent. The proportion of correct responses produced in the first half of the session was comparable to that achieved in the second half. Similarly, in both groups the three videos received comparable proportions of choices, and correct responses were equally frequent when the target-video appeared in the first, second or third place.

\section{Errors}

Most participants showed a consistent preference for movements recorded from one of the two distractorparticipants, which accounted for over $70 \%$ of total errors. This behavior was found in both groups (Control group: Cochran $Q_{(1)}=5.444, P<0.0196$; Imagery group: Cochran $\left.Q_{(1)}=3.600, P<0.005\right)$.

\section{Debriefing}

In a formal debriefing at the end of the session, all participants reported that judging an animation as being themselves felt somewhat 'bizarre', and some of them were uncertain about the overall accuracy of their responses. However, only four participants in each group defined the task as 'difficult'. Participants in the Imagery group tended to be more confident about their responses compared to those in the Control group (six of them expected to have responded correctly in over $60 \%$ of trials, whereas only three did in the Control group). Nearly all participants claimed to have used specific movement parameters as a cue to recognize their gesture (namely, its timing or velocity, 15 subjects). Six subjects in the Control group reported that on some occasions, they had tried to 'mentally visualize' their gestures in order to produce their response. Compared to the other participants, the number of correct identifications recorded for these subjects was increased by approximately $10-20 \%$.

\section{Discussion}

The results of Experiment 1 indicate that mentally rehearsing the action can facilitate recognition of one's own gesture. Sport psychology suggests that when simulating a movement we can adopt at least two kinds of strategies. First, we can imagine feeling as if we were actually executing a given action, thus attempting to experience the same kinesthetic feedback we get from the real movement (first-person perspective, internal imagery). Second, we can create a visual representation of the action and of the space where it takes place 
(third-person perspective, external imagery; Mahoney and Avener 1987). The term motor imagery usually refers to the first kind, and recent studies have emphasized the similarities that this form of imagery shares with movement execution. Imagined actions retain the same temporal characteristics as the corresponding real actions (Decety et al. 1989), and temporal regularities observed in motor acts (e.g., Fitts law) also appear in their imagined counterparts (Sirigu et al. 1995). Furthermore, comparable neural substrates likely support both mental simulation and motor execution (Decety et al. 1994; Stephan et al. 2002; Grafton et al. 1996; Roth et al. 1996; Gerardin et al. 2000).

In the present case, it was found that subjects' accuracy in detecting their motion patterns significantly increased following motor rehearsal, approaching that reported in on-line settings, i.e., when overt motor execution was required (Daprati et al. 1997; Franck et al. 2001). Considering the striking similarities that exist between motor imagery and movement execution (for a review, see Jeannerod and Frak 1999), this effect may depend on the reactivation of first person efferent information, which was removed by off-line settings. It could be assumed that by mentally simulating the action, participants made volitional and/or motor planning information about the gesture readily available. This in turn could have facilitated the operation of matching the observed movement with an internal representation of the same gesture. Accordingly, recognition of the correct motor pattern would be more easily prompted. This possibility would be in line with previous observations suggesting a specific effect of efferent information on action attribution (Tsakiris et al. 2005). Alternatively, one could suggest that by overtly referring to an internal representation of their movements, participants produced a visual image of their gesture, which in turn could have facilitated recognition. This second possibility would predict a robust influence of familiarity with one's visual motor pattern on action recognition. Specifically, observers would be more accurate in recognizing their gestures when the corresponding visual pattern is familiar.

Although a significant interaction between group and gesture was not found, the present results allow some cautious speculations on this issue. Here, imagery seemed to be particularly effective for the gesture of tracing a line, in agreement with the observation that the sight of one's hand holding a pen is very common. Yet, mental simulation seemed to improve accuracy also for recognition of finger walking, suggesting that motor imagery may be particularly helpful for movements devoid of rhythmic components. In fact, the two tapping movements received no benefit from mental rehearsal.
The specific roles of rhythmic cues and visual familiarity were examined in Experiment 2 and 3, respectively.

\section{Experiment 2}

In Experiment 2, we attempted to define which aspects of one's kinematics guide self-recognition. It can be assumed that the individual differences that occur during movement execution act as powerful cues in recognition. Variations in the motor pattern are more likely to emerge in complex gestures compared to stereotyped movements; hence, a series of gestures of increasing complexity was used in the present task. Differences in complexity were introduced by varying the contributions of three motion features: (a) rhythm, (b) degree of finger independence, and (c) posture familiarity. Information about hand morphology was removed by imposing the kinematics of each participant on a fixed hand template.

\section{Methods}

\section{Participants}

Ten new volunteers ( 7 females, 3 males; mean age $24.1 \pm 5.4$ years; education $15.3 \pm 1.9$ years) were recruited from among students at local university and nursing schools, and gave informed consent to participate in the experiment (as required by the local ethics guidelines, which comply with the 1964 Declaration of Helsinki). They had normal or corrected to normal vision and did not report previous history of neurological/psychiatric disorders. All were right-handed according to a shortened version of the Edinburgh Inventory (Oldfield 1971; mean laterality ratio $0.8 \pm 0.2$ ). Their demographic data did not differ from those of participants in Experiment 1 (according to separate Mann-Whitney $U$ tests performed on age, education, and laterality ratio between groups).

\section{Procedure}

The procedure was identical to that of the Control group in Experiment 1, except for four modifications, which concerned (a) gestures recorded, (b) form of the virtual hand, (c) interval between Sessions 1 and 2, and (d) modality of response. As for gestures, five movements were recorded, which were chosen based on (1) presence (or absence) of a rhythmic pattern in the movement; (2) degree of finger independence required by the gesture; (3) visual familiarity of the hand posture. In each of three gestures, one of these parameters 
was primarily represented. (1) In tapping with the index finger (IT, three times), rhythm was the most robust cue. (2) In index finger flexion (IF, once) finger independence (i.e., the capability to flex one finger independently) was more relevant. (3) In tracing a line (TL, thumb and index finger joined to grasp an imaginary piece of chalk, trace a line of the desired length and orientation), the effect of posture familiarity (i.e., the visual familiarity with the posture of one's hand when writing) was assumed to be most important. In two additional gestures, parameters were combined. In particular, (4) tapping with the hand (HT, hand taps three times) is both a familiar gesture, and a rhythmic movement, while (5) tapping with the fingers (FT, index, middle, ring and little finger tap individually on the table, once) adds to the previous features the additional requirement of finger independence. As such, it is likely to be the gesture providing the largest number of cues, i.e., the most complex of the five movements being studied.

Concerning the virtual hand, the 3D model of a hand (to which animation data were applied) was kept constant across participants, and elements linking the main hand joints were reduced $(\varnothing 5 \mathrm{~mm})$ to obtain a stick diagram. Distortions that might arise from original hand size were avoided by excluding individuals with exceedingly large or small hands, the present group varying by approximately $0.5 \mathrm{~cm}$ in the main hand/fingers dimensions. Variability in hand shape/size between individuals was thus minimized, except for postural components, which were purposefully retained. This latter aspect was controlled by selecting distractor-participants based on static and dynamic similarities with the observer's hand (see Appendix 1). The Recognition session took place 10-14 days after recording, to minimize memory effects. Participants pressed a numerical key to indicate which video they thought represented their own gesture. This allowed recording response latencies, in addition to accuracy measures.

\section{Design}

Information on elementary spatial and temporal parameters of the single gestures was used to properly select distractor-participants. In particular, participants whose motion parameters were temporally and spatially similar were grouped together, so that each observer always viewed movements that did not largely vary with respect to these features. This was done in order to present (to all participants) sets of three movements in which the difficulty of detecting differences could be considered substantially similar. As a result, any effect of movement type on the proportion of correctly recognized actions could be attributed specifically to richness of the cues provided by the type of movement presented (and not to a non-specific difficulty bias). Spatial and temporal parameters were analyzed as described in Appendix 1.

\section{Data analyses}

For each gesture, the proportion of correctly recognized movements and time to respond were collected for all participants. Only trials with procedural errors (less than 1\%) were excluded from the analyses. Response latencies were computed as the interval elapsed between the end of the third video and the subject's response. As this value was considered basically as an index of the time required to reach a decision, incorrect responses were not discarded. Average duration of each video-clip was $2.7 \pm 0.3 \mathrm{~s}$; average duration of video presentation was $8.0 \pm 0.3 \mathrm{~s}$. Means of correct responses and response latencies for the five gestures were compared by means of two separate 1-way ANOVAs. To control for the effects of a skewed distribution and satisfy the conditions for parametric statistical test, proportions of correct responses and response times were first submitted to arcsine and logarithmic transformation, respectively. The Scheffè test was used for post-hoc analyses.

\section{Results}

\section{Accuracy}

Participants correctly chose the video corresponding to their movement in $65 \%$ of trials (overall proportion of correct detections $0.65 \pm 0.30$ ). Despite the less informative hand reconstruction, the proportion of correctly recognized gestures in the present experiment matched that of the Control group in Experiment 1 (a 2-way ANOVA comparing responses of the three gestures that were common to both experiments confirmed the absence of any significant difference between groups). Identification of the correct movement occurred significantly above chance level for all gestures, except for IF (IT: $\chi^{2}=35.67$; HT: $\chi^{2}=60.79$; FT: $\chi^{2}=62.42$; LT: $\chi^{2}=45.92$, all $\left.P<0.001\right)$, and the proportion of correct detections clearly varied according to gesture (see Fig. 3, left panel). The highest recognition rate was found in the FT $(0.80 \pm 0.28)$ and in the HT conditions $(0.79 \pm 0.23)$. Recognition rate was lower for the other gestures (LT: $0.64 \pm 0.35$; IT: $0.60 \pm 0.29)$, and lowest for IF $(0.43 \pm 0.22)$. Accordingly, a 1-way ANOVA showed a significant main 
Fig. 3 Percentage of correctly recognized gestures (left pan$e l$ ), and latency to provide a response (in $\mathrm{ms}$, right panel) for the movements tested in Experiment 2. Whiskers report standard error of the mean. Abbreviations on $x$-axis indicate gestures: $I F$ index finger flexion, IT tapping with the index finger, $T L$ trace a line, $H T$ tapping with the hand, FT tapping with the fingers

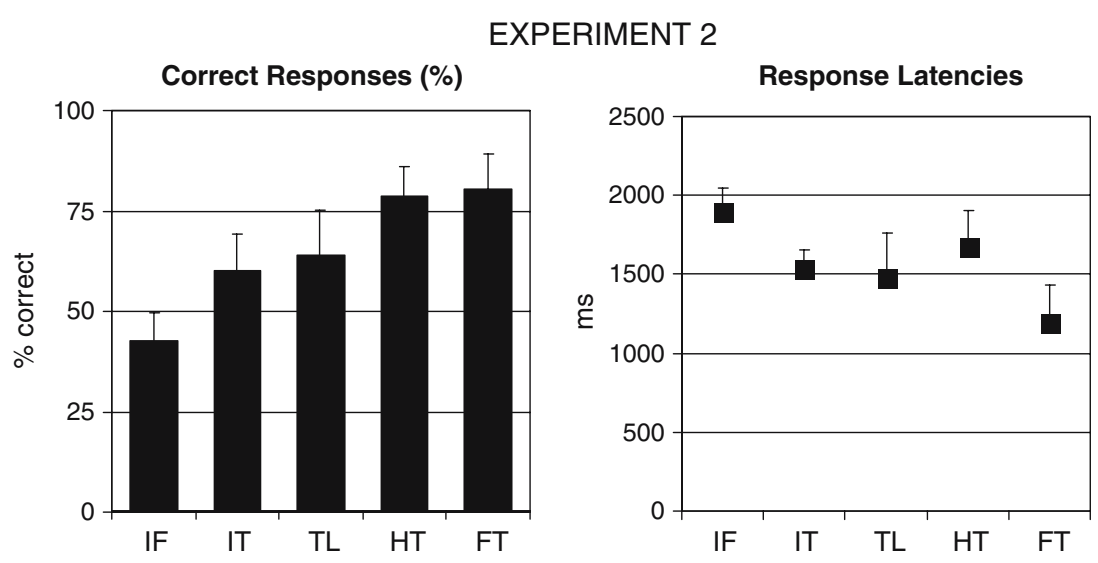

effect of gesture $\left(F_{(4,36)}=3.531 ; P=0.0157\right)$. Post-hoc analysis pointed out a significant difference between correct detections achieved for FT and IF $(P=0.042)$.

As in Experiment 1, learning effects were not apparent. The proportion of correct responses produced in the first half of the session was comparable to that in the second half. Similarly, all videos received comparable proportions of choices, and correct responses were equally frequent when the target-video appeared in the first, second or third place.

\section{Latency}

Participants required longer decision times when watching simple gestures, such as IF $(1897.7 \pm 437 \mathrm{~ms})$ compared to complex ones, i.e., FT (1188.5 $\pm 706 \mathrm{~ms})$. This observation was confirmed by a 1-way ANOVA $\left(F_{(4,32)}=3.363 ; P=0.021\right.$; see also Fig. 3 , right panel $)$. In accordance with the distribution of correct responses, post-hoc analysis pointed out a significant difference between the shorter latencies required to reach a decision for FT and the longer latencies required for $\operatorname{IF}(P=0.036)$.

\section{Errors}

As reported in the previous experiment, when mistaken, almost all participants showed a consistent preference for one of the two distractor-participants, which accounted for over $70 \%$ of total errors (Cochran $\left.Q_{(1)}=6.400, P<0.0114\right)$.

\section{Debriefing}

In a formal debriefing at the end of the session, more participants reported the task as difficult (six participants) compared to Experiment 1, possibly due to the fact that the virtual hand was less informative (i.e., devoid of morphological cues). Yet, most of them were quite confident about the accuracy of their performance (only three subjects expected to have scored less than $50 \%$ correct responses). All reported that on several occasions, they had 'imagined' executing the movement in order to produce their response. Nearly all participants claimed to have used specific movement parameters as a cue to recognize their gesture (namely, its timing or velocity), although most of them reported that they often simply 'knew' their movement when it appeared on the screen.

\section{Discussion}

The most striking result in the present experiment was the effect of gesture complexity, which strongly suggests that the degree to which individual differences in motor execution can be expressed represents the critical factor in recognition of one's motion patterns. Indeed, even simple gestures, such as those examined here, involve complex changes in several spatio-temporal parameters having multiple degrees of freedom. Some kinematic features of human movements tend to be invariant across individuals. They include bellshaped velocity profiles, the two-thirds power law, maximum smoothness, and linear inter-segmental coordination (Jeannerod 1988; Lacquaniti 1989). Based on these observations, it can be assumed that the more simple the movement, the less variable its execution across individuals and consequently, the less evident the differences associated with it. In addition, the particular features of the selected gestures allow speculations on the nature of the cues that made selfrecognition possible. It should be recalled here that familiar static cues such as shape and size were not available, thus forcing observers to rely on motionrelated cues.

Results suggest that participants used a combination of cues rather than relying on a single kinematic feature. Based on the proportion of correct responses, we 
can assume that familiar postural details are not as helpful as the opportunity to use the information offered by rhythm and knowledge of ones' finger independence. Indeed, participants were more accurate when recognizing their movements in FT (where these cues were combined) compared to TL, despite the frequent observation of one's hand in writing, which could have prompted recognition based on visual familiarity. Similarly, the capability to independently move each finger (i.e., finger independence) is certainly a major characteristic of an individual's movement, but likely carries only part of the information. IF, which strongly depends on finger independence, induced a significantly larger proportion of errors compared to FT, where this component is combined with rhythmic information.

It could be argued here that rhythm may represent the most reliable cue to action recognition. This observation would be consistent with previous findings demonstrating that subjects can discriminate between their performance and that of another participant when acoustic traces of their clapping hands are substituted by uniform tones that retain the original rhythm and tempo (Flach et al. 2004). The present results extend this observation, showing that when visual motor traces are being judged, rhythm can be an effective cue as well. However, it was clearly not the only one. If temporal cues had been sufficient for self-recognition, we should have obtained an equal number of correct recognitions for the three types of tapping movements analyzed in the present experiment. In contrast, despite comparable rhythmic components, IT did not produce as many correct responses as HT or FT, in which more than one cue was available (the difference was significant at t-tests comparing the tapping gestures, $P<0.05)$. Stated in a different way, accuracy increased for movements that allowed for larger interindividual variability in the execution, because of the major number of relevant parameters.

\section{Experiment 3}

In Experiment 3, we explored the role played in selfrecognition by visual familiarity with one's movement. We argued that one possible reason why self-generated actions are easily recognizable may be the extensive visual experience we have with them in daily life, and which could be reinforced in laboratory conditions. To test this hypothesis, we introduced two manipulations, either in the recording session or in the recognition phase. In the former, we removed visual episodic memory information by recording movements in a fully darkened room (NoVision Group). In the latter, we reduced visual familiarity with the dynamic aspects of the movement by playing animation data at either half or twice their normal pace (Speed Group).

Methods

\section{Participants}

Seven new volunteers recruited from the local university and nursing school, participated in the present experiment (NoVision Group, seven females; mean age $24.0 \pm 2.9$ years; education $16.7 \pm 1.7$ years, handedness ratio $0.8 \pm 0.2$ ). These new participants did not differ from those in Experiment 2 in demographic measures, according to separate Mann-Whitney $U$ tests performed on age, education, laterality ratio and days elapsed between first and second session. They signed informed consent to participate (as required by the local ethics guidelines, which comply with the 1964 Declaration of Helsinki). In addition, the same ten volunteers involved in Experiment 2 (Speed Group) were enrolled also in the present experiment. To avoid fatigue, they returned to the lab the next day to perform this second recognition task.

\section{Procedure}

The procedure was identical to that of Experiment 2, except for two aspects that aimed to reduce visual familiarity. For volunteers who had not participated in the previous experiment (NoVision Group), the recording session took place in the dark, i.e., the light was switched off in the lab, soon after participants had been shown an example of the gestures they were required to execute. A tactile guide was placed over the table to guarantee that participants maintained the hand in the correct location during the task. The recognition session was identical to that of Experiment 2 (i.e., the videos were played back at the normal speed). To select distractors for each of the seven participants in this NoVision Group, the same procedure as described in Experiment 2 was applied (see Appendix $1)$. For volunteers who had previously participated in Experiment 2 (Speed Group), a second recognition session was run in which movements of the virtual hand played at half (slow trials) or twice (fast trials) the original speed. Thus, rhythmic components (if present) were maintained, but the overall tempo was modified. To ensure that the total duration of video presentations was comparable, fast and slow videos were pseudo-randomly distributed within trials (i.e., to 
account for the fact that fast videos were shorter than slow ones). The different combinations of fast and slow videos thus obtained were counterbalanced across blocks. The average duration for each set of three videos was $10.68 \pm 0.69 \mathrm{~s}$. The same number of fast and slow trials was presented in each block in random order.

\section{Data analyses}

Data analyses were identical to those of Experiments 1 and 2. Only trials with procedural errors (less than $1 \%$ ) were excluded from the analyses. For participants in the NoVision Group, only proportion of correct detections was recorded. Means of correct responses for the five gestures were compared by means of 1-way ANOVA. For the Speed Group, proportions of correctly recognized gestures (and corresponding latencies) for fast and slow trials were computed separately, and the corresponding means were analyzed by a 2-way ANOVA (within-subject factors were Speed: fast vs. slow and Gesture: 5 levels).

Measures collected in Experiments 2 and 3 were compared by means of separate 2-way ANOVAs.

\section{Results}

\section{Visual feedback manipulation}

Participants in the NoVision group produced approximately $65 \%$ correct responses (overall proportion of correct detections, $0.661 \pm 0.2$ ). Accuracy did not differ significantly from that reported in Experiment 2 and varied similarly as a function of gesture complexity (see Fig. 4). Tapping movements were more easily recognized compared to simple gestures: a 2-way ANOVA comparing the five movements in the two experiments showed the significant main effect of Gesture $\left(F_{(4,60)}=3.927 ; P<.0067\right)$. The main effect of Group was non significant, as was the interaction.

\section{Speed manipulation}

Manipulation of speed of video-presentation did not modify the rate of correct self-detections. Participants still produced approximately $65 \%$ correct responses (overall proportion of correct detections $0.66 \pm 0.36$ ). Accuracy did not significantly differ from that recorded in Experiment 2. When the effect of Speed is specifically considered, comparable proportions of correct detections were found for slow $(0.67 \pm 0.34)$ and fast trials $(0.65 \pm 0.38)$. All movements were equally well recognized, in both fast and slow trials. A 2-way

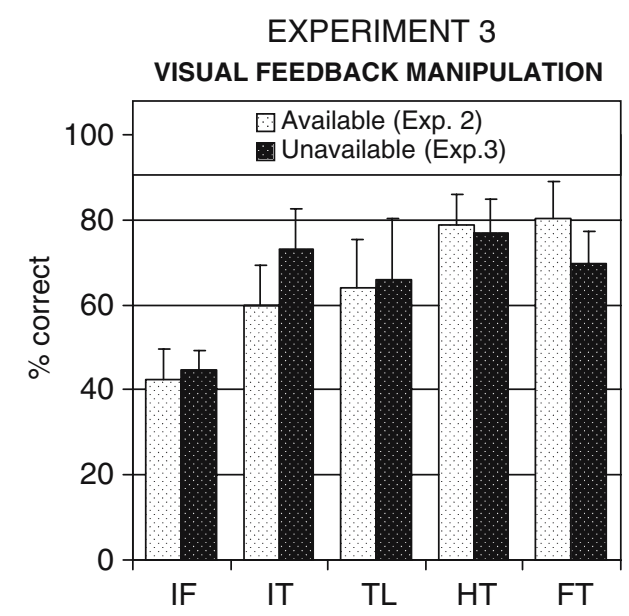

Fig. 4 Percentage of correctly recognized gestures for the movements tested in Experiment 3. Visual Feedback manipulation (recording session). Whiskers represent standard error of the mean. Abbreviations on $x$-axis indicate gestures: $I F$ index finger flexion, $I T$ tapping with the index finger, $T L$ trace a line, $H T$ tapping with the hand, $F T$ tapping with the fingers

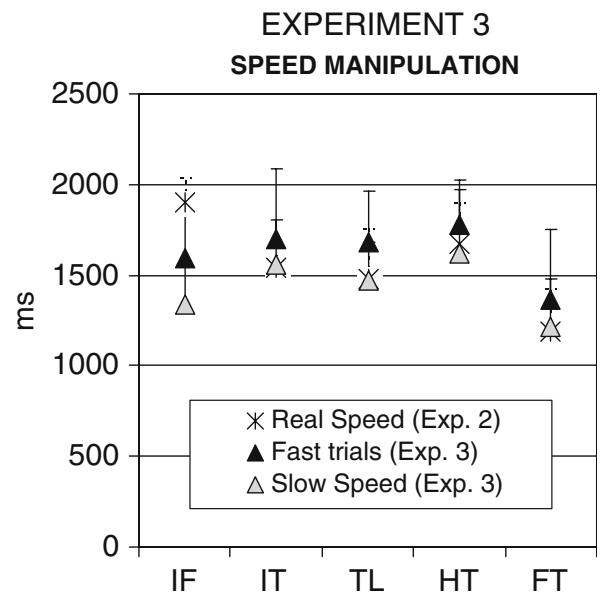

Fig. 5 Latency to provide a response (in $\mathrm{ms}$ ) for the movements tested in Experiment 3. Speed manipulation (recognition session). Whiskers represent standard error of the mean. Abbreviations on $x$-axis indicate gestures: $I F$ index finger flexion, IT tapping with the index finger, $T L$ trace a line, $H T$ tapping with the hand, FT tapping with the fingers

ANOVA comparing the five gesture in fast and slow presentation showed no significant differences in proportion of correct detections $\left(F_{(4,36)}=1.285 ; P<0.294\right)$. A significant main effect of Gesture was found only for response latencies $\left(F_{(4,36)}=3.065 ; P<0.028\right.$, see also Fig. 5), finger tapping leading to significantly faster responses compared to the remaining gestures. Following speed manipulation, a consistent preference for one of the two distractor-participants was no longer found. 
Discussion

Removal of episodic visual memory of the performed action (by recording in a fully darkened room) did not significantly modify accuracy and latencies of subjects' responses, suggesting that familiarity with the visual appearance of one's motion makes a minor contribution to recognition processes. However, this result could also reflect the fact that, although visual information was removed in the laboratory setting, participants may have retained some visual memory of the most common gestures, such as tracing a line or tapping. Two observations rule out this interpretation. First, had visual memory played a critical role, one should have expected the act of tracing a line to be judged accurately by participants, due to the extreme familiarity of our hand posture while holding a pen. This was not the case (see Figs. 3, 4). Second, modification of the familiar speed of video presentations did not affect accuracy and latency of subjects' responses.

This second result has a series of interesting implications. On the one hand, it suggests that, regardless of the overall change in tempo, participants still correctly recognized their movement based on comparable cues, as shown by the fact that the distributions of their response was the same (see Figs. 4, 5). On the other hand, this result apparently contrasts with previous findings on clapping (Flach et al. 2004). There, it was found that, if subjects listened to beep sequences that retained the relative timing of their own clapping, but were played in the tempo produced by another participant, they lost the ability to identify their own clapping. The apparent inconsistency of these results may be due to the availability, in the present visual case, of spatial cues, which were unaffected by speed modifications. Summing up, the present observations point towards the possibility that, notwithstanding a potential role played by visual familiarity in everyday situations, selfrecognition can be gained from cues that do not depend on familiarity.

\section{General discussion}

The present research investigated the differential contributions of three sets of cues produced by motor activity to self-recognition: (a) first-person efferent information; (b) individual differences in one's kinematics, as a function of gesture complexity; (c) visual familiarity with one's movements. The results can be summarized as follows. (1) Overtly referring to the internal representation of one's gesture, by means of motor imagery, facilitates self-recognition. (2) Self-rec- ognition benefits from gesture complexity, in terms of interval timing and/or finger independence (the richer the gesture, the more accurate its recognition). (3) Familiarity with the visual outcome of one's motion does not represent a major cue to self-recognition. Taken together, these results suggest that, in the absence of morphological cues, kinematic templates largely suffice for self-recognition.

By what mechanism do kinematic features permit correct self-identification? Echoing what has been described in face recognition (Bruce and Young 1986), we suggest that accurate identification of one's motion must take into account both invariant and changeable aspects of the movement. The former aspect, being related to human anatomical and biomechanical constraints, should be similarly represented across individuals. As such, it likely represents the substrate for entirely sharable motor representations, which enable us to infer the meaning of other's actions by the resonance produced within our motor system, or by the concurrent simulation of the same action (see Blakemore and Decety 2001 for a review). In contrast, changeable aspects would arise from how these invariants are dealt with by each person, thus creating the variability in motion patterns that may represent the basis for recognizing individuals. Very little is known about individual differences in kinematics. The results of the present research suggest that they arise from complex associations of multiple motor variables, rather than from modulation of single kinematic parameters. Although speculative, it could be assumed that also non-motor aspects, such as emotion, personal history, or mechanisms related to social communication could contribute to characterize an individual's motor style. These changeable aspects of the movement would pertain more specifically to the agent of the action. As such, they could prompt self-recognition by inducing greater resonance in the observer's motor system, or permitting a more veridical simulation. The existence of close similarities between motor representations and overt motor activity has largely been proved (for a review, Jeannerod 2001), leading to the hypothesis that action perception and action execution may actually share a common coding (see Knoblich and Flach 2003 for a review). The existence of a mirror system in both monkeys and humans provides neurophysiological support for this idea (for a review, Rizzolatti et al. 2002; Buccino et al. 2004). Along these lines it can been assumed that when observing one's past actions, stronger similarities emerge between the perceptual codes provided by the observed movement and the subject's internal motor codes, similar to what happens when individuals attend to auditory events that are the products of their actions (Repp and Knoblich 2004). The 
results of the present study further stress the role that kinematic features play within this process.

Another interesting finding is the beneficial effect produced on accuracy in self-recognition by motor imagery. This result has two interesting implications: on the one hand, it confirms that the process of mental simulation actually retains the precise features that characterize one's movement. On the other hand, it indicates that observers spontaneously engage in a form of kinesthetic, rather than visual, imagery when rehearsing their own actions. Indeed, visual familiarity was shown to play a minor role in self-recognition. Hence, we cannot ascribe the facilitating effect of imagery to activation of a visual image of one's own gesture. Given the critical role played by efferent information in self-recognition (Tsakiris et al. 2005), it appears that simulating the action helped participants to reactivate those first-person cues that the off-line setting otherwise had removed. This could happen only if the observer spontaneously engaged in kinesthetic motor imagery. It is interesting to note here that the participants in the present study were not high imagers according to the MIQ (Hall and Pongrac 1983), but scored mainly as medium to low imagers. This observation suggests that the form of kinesthetic imagery involved here is probably quite elementary, i.e., belongs to a comparable extent to all individuals, and primarily recruits intentional and/or volitional processes. We can speculate that the voluntary act of "preparing" for the action may in turn activate the corresponding kinesthetic representations, facilitating self-recognition, in spite of a modest ability to properly mentally portray the action on the part of the observer.

Finally, it should be noted that whenever possible, we selected distractor-participants to match the elementary spatial and temporal parameters of the observer's movement. Hence, with a reasonable error margin, the movements provided as alternatives were expected to be equivalent to the eye of the observer, who should have chosen either one with equal probability. This was not found, since when in error, participants showed a consistent "preference" for one alternative. Given the limited number of errors produced in the task, a systematic analysis could not be run, to identify the processes guiding the preference. Yet, the available observations suggest that the participants always selected the movements of the person that shared the particular combination of motorrelated cues that characterized her own gesturing. This was observed specifically for tapping movements. When a clear temporal pattern could be identified in the target subject, such as a progressive increase of inter-tap interval duration from the first to the last tap of the series, the same pattern was found only in the preferred alternative. The same was true for those participants consistently showing a progressive increase (or decrease) of movement amplitude in the tapping gestures. It is interesting to note that participants were largely unaware of these peculiarities in their error distribution. We propose that a 'borderline area' may exist, where a sense of recognition emerges in the absence of correct identification. This phenomenon could be responsible for the systematic misidentifications found in the experiments reported here, suggesting that individual differences may emerge at various levels of complexity.

Acknowledgments The authors are grateful to Dr. M. Carrozzo for support in setting up the motion capturing procedure, L. Granjon and V. Spurio for their help in preparing the virtual hand and presentation software, and Prof. G. Rizzolatti for discussing the data. The authors wish to thank B. Repp and an anonymous referee for editing and commenting on an earlier draft of the manuscript. The financial support of Italian University Ministry (PRIN and FIRB projects), Italian Health Ministry and Italian Space Agency is gratefully acknowledged.

\section{Appendix 1}

Analyses of kinematic parameters and selection of distractor-participants

Spatial and temporal parameters were analyzed as follows. The time course of the displacement of the markers placed on the fingertips was used to compute the beginning and end of each movement. The single data points were plotted and the waveform of each movement was analyzed by detecting all peaks of the function. Movement start was defined as the sample corresponding to the origin of the rising slope of the first peak, where the difference between two successive points significantly exceeded a constant noise factor (0.001) for the relevant marker (I4 for IT, IF and TL; the earliest finger-tip marker to show a stable displacement for HT and FT). Likewise, the temporal sample corresponding to the end of the falling slope of the last abovenoise peak defined the end of the movement. Amplitudes (and angles) were defined on the basis of markers' displacement compared to the plane of the table for the detected peaks. For all gestures, total movement time (time difference between the start and end of the movement) was computed. For IF, maximal angle of finger flexion was also analyzed. For the three tapping gestures, inter-tap intervals and maximal amplitude of hand/finger lift were computed. For TL, length of the line, peak velocity of the displacement of the marker on 
Table 1 Means and SD for main spatial and temporal parameters recorded in Experiment 2 (as a measure of overall variability in gesture execution for the group)

\begin{tabular}{|c|c|c|c|c|c|}
\hline \multirow{2}{*}{$\begin{array}{l}\text { Gesture } \\
\text { Index tapping }\end{array}$} & \multicolumn{2}{|l|}{ Movement parameters } & \multirow{2}{*}{$\begin{array}{l}\text { Gesture } \\
\text { Index flexion }\end{array}$} & \multicolumn{2}{|l|}{ Movement parameters } \\
\hline & Total movement time (s) & $1.40 \pm 0.30$ & & Total movement time (s) & $0.82 \pm 0.16$ \\
\hline & Inter-tap interval (s) & & & $\begin{array}{l}\text { Prox. Interphalangeal } \\
\text { angle (deg) }\end{array}$ & $79.44 \pm 11.5$ \\
\hline & 1st tap & $0.13 \pm 0.06$ & & & \\
\hline & 2nd tap & $0.12 \pm 0.5$ & & & \\
\hline & Max. amplitude (mm) & & & & \\
\hline & 1st tap & $3.97 \pm 1.06$ & & & \\
\hline & 2 nd tap & $3.99 \pm 1.14$ & & & \\
\hline & $3 r d$ tap & $4.21 \pm 1.14$ & & & \\
\hline \multirow[t]{8}{*}{ Hand tapping } & Total movement time (s) & $1.58 \pm 0.37$ & Trace a Line & Total movement time (s) & $1.51 \pm 0.47$ \\
\hline & Inter-tap interval (s) & & & $\begin{array}{l}\text { Maximal displacement } \\
(\mathrm{mm}, \text { length of the line) }\end{array}$ & $23.48 \pm 6.62$ \\
\hline & 1st tap & $0.13 \pm 0.06$ & & Peak velocity of index & $71.38 \pm 27.04$ \\
\hline & 2 nd tap & $0.12 \pm 0.05$ & & fingertip (mm/s) & \\
\hline & Max. amplitude (mm) & & & Pinkie elevation (mm) & $2.20 \pm 1.49$ \\
\hline & 1st tap & $5.41 \pm 2.30$ & & & \\
\hline & 2nd tap & $4.98 \pm 2.03$ & & & \\
\hline & 3rd tap & $5.41 \pm 2.27$ & & & \\
\hline \multirow[t]{5}{*}{ Finger tapping } & Total movement time (s) & $2.28 \pm 0.52$ & Finger tapping & Max. amplitude (mm) & \\
\hline & Inter-tap interval (s) & & & 1 st tap & $4.33 \pm 1.90$ \\
\hline & 1st tap & $0.19 \pm 0.12$ & & 2nd tap & $4.05 \pm 2.02$ \\
\hline & 2nd tap & $0.15 \pm 0.24$ & & 3 rd tap & $3.59 \pm 2.00$ \\
\hline & 3rd tap & $0.05 \pm 0.33$ & & 4 th tap & $3.07 \pm 1.57$ \\
\hline
\end{tabular}

Values measured for single subjects were used to select distractors whose mean movement parameters ranged within 2 SD from those of the target-subject on at least four gestures

the index fingertip and elevation of the little finger on the plane of the table were measured. Means and variability across subjects for the analyzed parameters for the five gestures are reported in Table 1.

Based on this information, distractors for each participant were selected according to four constraints. First, main hand/finger dimensions differed by less than $0.5 \mathrm{~cm}$. Second, for each gesture and for at least a half of the analyzed parameters, mean values recorded from the distractors' movements were within a tolerance interval equal to the range defined by the observer's movements. Third, mean values recorded from the distractors' movements never exceeded this range by more than $25 \%$ for the remaining parameters. Finally, this was true for at least four out of five gestures.

\section{References}

Beardsworth T, Buckner T (1981) The ability to recognize oneself from a video recording of one's movements without seeing one's body. Bull Psychon Soc 18:19-22

Blakemore S-J, Decety J (2001) From the perception of action to the understanding of intention. Nat Rev Neurosci 2:561-567

Bruce V, Young A (1986) Understanding face recognition. Br J Psychol 77:305-327

Buccino G, Binkofski F, Riggio L (2004) The mirror neuron system and action recognition. Brain Lang 89:370-376
Corlett J T, Anton J, Kozub S, Tardiff M (1989) Is locomotor distance estimation guided by visual imagery? Percept Mot Skills 69:1267-1272

Daprati E, Sirigu A (2005) Action recognition disorders following parietal damage. In: Freund H-J, Jeannerod M, Hallet M, Leiguarda R (eds) Higher-order Motor Disorders - From neuroanatomy and neurobiology to clinical neurology. Oxford University Press, Oxford

Daprati E, Franck N, Georgieff N, Proust J, Pacherie E, Dalery J, Jeannerod M (1997) Looking for the agent: an investigation into consciousness of action and self-consciousness in schizophrenic patients. Cognition 65:71-86

Decety J, Jeannerod M, Prablanc C (1989) The timing of mentally represented actions. Behav Brain Res 34:35-42

Decety J, Perani D, Jeannerod M, Bettinardi V, Tadary B, Woods R, Mazziotta JC, Fazio F (1994) Mapping motor representations with positron emission tomography. Nature 371:600 602

Flach R, Knoblich G, Prinz W (2004) Recognizing one's own clapping: The role of temporal cues. Psychol Res 69:147-156

Franck N, Farrer C, Georgieff N, Marie-Cardine M, Dalery J, d'Amato T, Jeannerod M (2001) Defective recognition of one's own actions in patients with schizophrenia. Am J Psychiatry 158:454-459

Gerardin E, Sirigu A, Lehericy S, Poline JB, Gaymard B, Marsault C, Agid Y, Le Bihan D (2000) Partially overlapping neural networks for real and imagined hand movements. Cereb Cortex 10:1093-1104

Grafton ST, Arbib MA, Fadiga L, Rizzolatti G (1996) Localization of grasp representations in humans by positron emission tomography 2: observation compared with imagination. Exp Brain Res 112:103-111

Hall C, Pongrac J (1983) Movement imagery questionnaire. University of Western Ontario, London Ontario 
Jeannerod M (1988) The neural and behavioural organization of goal-directed movements. Oxford University Press, Oxford

Jeannerod M (2001) Neural simulation of action: a unifying mechanism for motor cognition. Neuroimage 14:S103-S109

Jeannerod M, Frak V (1999) Mental imaging of motor activities in humans. Curr Opin Neurobiol 9:735-739

Johansson G (1973) Visual perception of biological motion and a model for its analysis. Percept Psychophys 14:201-211

Knoblich G, Flach R (2003) Action identity: evidence from selfrecognition prediction and coordination. Conscious Cogn 12:620-632

Knoblich G, Prinz W (2001) Recognition of self-generated actions from kinematic displays of drawing. J Exp Psychol Hum Percept Perform 27:456-465

Kozlowski LT, Cutting JE (1977) Recognizing the sex of a walker from point-lights display. Percept Psychophys 21:575-580

Lacquaniti F (1989) Central representations of human limb movement as revealed by studies of drawing and handwriting. Trends Neurosci 12:287-291

Loula F, Prasad S, Harber K, Shiffrar M (2005) Recognizing people from their movement. J Exp Psychol Hum Percept Perform 31:210-220

Mahoney MJ, Avener M (1987) Psychology of the elite athlete: an explorative study. Cognit Ther Res 1:135-141

Nielsen TI (1963) Volition: a new experimental approach. Scand J Psychol 4:225-230
Oldfield RC (1971) The assessment and analysis of handedness: the Edinburgh inventory. Neuropsychologia 9:97-113

Repp BH, Knoblich G (2004) Perceiving action identity: how pianists recognize their own performances. Psychol Sci 15:604-609

Rizzolatti G, Fogassi L, Gallese V (2002) Motor and cognitive functions of the ventral premotor cortex. Curr Opin Neurobiol 12:149-154

Roth M, Decety J, Raybaudi M, Massarelli R, Delon-Martin C, Segebarth C, Morand S, Gemignani A, Decorps M, Jeannerod M (1996) Possible involvement of primary motor cortex in mentally simulated movement: a functional magnetic resonance imaging study. Neuroreport 7:1280-1284

Sirigu A, Cohen L, Duhamel JR, Pillon B, Dubois B, Agid Y (1995) A selective impairment of hand posture for object utilization in apraxia. Cortex 31:41-56

Sirigu A, Daprati E, Pradat-Diehl P, Frank N, Jeannerod M (1999) Perception of self-generated movement following left parietal lesion. Brain 122:1867-1874

Stephan KM, Fink GR, Passingham RE, Silbersweig D, CeballosBaumann AO, Frith CD, Frackowiak RS, van den Bos E, Jeannerod M (2002) Sense of body and sense of action both contribute to self-recognition. Cognition 85:177-187

Tsakiris M, Haggard P, Franck N, Mainy N, Sirigu A (2005) A specific role for efferent information in self-recognition. Cognition 96:215-231

Wolff W (1943) The expression of personality. Harper, New York 